TRANSACTIONS OF THE

AMERICAN MATHEMATICAL SOCIETY

Volume 355, Number 6, Pages 2161-2182

S 0002-9947(03)03161-1

Article electronically published on January 31, 2003

\title{
CENTRAL KÄHLER METRICS
}

\author{
GIDEON MASCHLER
}

\begin{abstract}
The determinant of the Ricci endomorphism of a Kähler metric is called its central curvature, a notion well-defined even in the Riemannian context. This work investigates two types of Kähler metrics in which this curvature potential gives rise to a potential for a gradient holomorphic vector field. These metric types generalize the Kähler-Einstein notion as well as that of Bando and Mabuchi (1986). Whenever possible the central curvature is treated in analogy with the scalar curvature, and the metrics are compared with the extremal Kähler metrics of Calabi. An analog of the Futaki invariant is employed, both invariants belonging to a family described in the language of holomorphic equivariant cohomology. It is shown that one of the metric types realizes the minimum of an $L^{2}$ functional defined on the space of Kähler metrics in a given Kähler class. For metrics of constant central curvature, results are obtained regarding existence, uniqueness and a partial classification in complex dimension two. Consequently, on a manifold of Fano type, such metrics and Kähler-Einstein metrics can only exist concurrently. An existence result for the case of non-constant central curvature is stated, and proved in a sequel to this work.
\end{abstract}

\section{Introduction}

Of the many curvature notions in higher-dimensional Riemannian geometry, scalar curvature holds one claim to simplicity, by virtue of being a generalization of the Gauss curvature which is still a mere function on the given space. This work investigates another such curvature potential, which we term the central curvature. Although the notion belongs in the Hermitian, or even Riemannian arena (cf. [La]), we confine ourselves to Kähler geometry, in the context of the search for distinguished metrics on compact manifolds.

Classically, the scalar curvature is viewed as the trace of a linear map, namely the Ricci endomorphism. Correspondingly, the central curvature is its determinant. Since the Ricci form on a Kähler manifold is determined by the volume form, according to the formula

$$
\rho=-i \partial \bar{\partial} \log \operatorname{det} g
$$

it is perhaps not surprising that the central curvature potential may be employed for the consideration of volume-decreasing holomorphic maps between manifolds with negative Ricci form [Kb3. What is perhaps less expected, is that much of the

Received by the editors November 1, 1999.

2000 Mathematics Subject Classification. Primary 53C55, 53C25, 58E11.

Partially supported by the Edmund Landau Center for research in Mathematical Analysis and Related Areas, sponsored by the Minerva Foundation (Germany). 
theory of Kähler metrics with distinguished scalar curvature has a central curvature counterpart.

For example, the announcement BM1 describes, even in the more general Hermitian context, a functional whose critical points are metrics with constant central curvature. This is the analog of the $K$-energy map used for metrics of constant scalar curvature.

More fundamental to our approach is the appearance of a cohomological invariant $\mathcal{B}$, a character on the Lie algebra of holomorphic vector fields. Its non-vanishing provides an obstruction to the existence of metrics of constant central curvature (cf. $[\mathrm{FT}]$ ). For metrics of constant scalar curvature, the associated invariant $\mathcal{F}_{[\omega]}$ is the well-known Futaki invariant [Ft1, [Ft3]. The latter, however, obstructs existence only in a fixed Kähler class $[\omega]$. It was originally introduced for $[\omega]=c_{1}$, i.e., when the Kähler class is the first Chern class of a manifold of Fano type. In this context it provides an obstruction to the existence of Kähler-Einstein metrics. For the latter, both the scalar and central curvatures are constant, and indeed it was shown in [FR1, [FR2] that, in this setting, $\mathcal{F}_{c_{1}}=\mathcal{B}$. This gave an additional form for the Futaki invariant, which was systematically exploited to relate it with various secondary characteristic invariants. However, the definition of $\mathcal{B}$, unlike that of $\mathcal{F}_{c_{1}}$, also shows that this invariant contains information pertaining to metrics in all Kähler classes. Moreover, $\mathcal{B}$ is well-defined even for manifolds which are not of Fano type. Both $\mathcal{B}$ and $\mathcal{F}_{\omega}$ belong to a family of invariants associated with the elementary symmetric functions of the Ricci endomorphism, and are treated in this work within the framework of holomorphic equivariant cohomology $[\mathrm{Cr},[\mathrm{Lu}],[\mathrm{Z}]$.

As it will be shown that the existence problem regarding metrics of constant central curvature is largely settled through Yau's resolution of the Calabi conjecture $[\mathrm{Yu}$, it is important to note that $\mathcal{B}$ is significantly related even to metrics for which the central curvature is not necessarily constant, but instead is a holomorphy potential of a gradient holomoprhic vector field. We call both the metric and the vector field central. The analogous definitions using the scalar curvature give the extremal metrics and vector fields of Calabi C1], C22. These were originally defined using a variational principle, but the associated Euler-Lagrange equation leads, on a compact manifold, to a distinguished gradient holomorphic vector field, with the scalar curvature serving as the holomorphy potential.

If an extremal metric has a non-trivial extremal vector field, the Futaki invariant cannot vanish on it. More importantly, there exists a duality pairing which intertwines this functional with the extremal vector field [C2], [FM], [H2]. This pairing is realized by a non-degenerate bilinear form on a subalgebra of the gradient holomorphic vector fields, and is also a Kähler class invariant [FM]. Moreover, a second natural invariant bilinear form may be used, and then the resulting duality associates with the Futaki invariant a vector field which is not the extremal one, but rather the distinguished vector field of a Kähler-Ricci soliton (or quasi-Einstein metric).

Pairing the invariant $\mathcal{B}$ with each of the two bilinear forms also gives two types of distinguished vector fields, and therefore two types of (central) metrics, differing by whether the central curvature, or rather its Laplacian, constitutes the holomorphy potential. Metrics of constant central curvature are, of course, associated to the trivial vector field, and thus belong to both types. 
Although this outline and a sizable part of this work demonstrate that the theories of extremal and central metrics proceed, to a large degree, in parallel to one another, there appear to be marked differences with regard to existence and uniqueness. For example, it is not known whether the existence of an extremal metric in one Kähler class implies the existence of such metrics throughout the cohomology cone of Kähler classes. Examples are known for which this either does not hold, or else uniqueness within a particular Kähler class fails [Tf]. In contrast, whenever they exist, central metrics of constant central curvature do fill the entire Kähler cone. They also have good uniqueness properties, at least on manifolds that admit non-Ricci-flat Kähler-Einstein metrics. In fact, on a manifold of Fano type, the existence of such a metric implies the existence of a Kähler-Einstein metric.

On the other hand, it is fairly clear that central metrics exist on fewer manifolds than extremal metrics. For example, all Hirzebruch surfaces admit extremal metrics, whereas, at least within the framework of a particular ansatz, we can show that only the first one admits a central metric of the first type, of non-constant central curvature [HM]. Moreover, at least for the case of nowhere-vanishing central curvature, the existence of such a metric is tied with particular asymptotic growth rates of all dimensions of the sheaf cohomology groups associated with (powers of) the anticanonical line bundle. The corresponding relation for metrics with nowhere-vanishing scalar curvature is much weaker.

We proceed to describe the content of the various sections of this work. Central curvature, metrics and vector fields are defined in Section 2, and compared with scalar curvature and extremal metrics. Section 3 gives a summary of holomorphic equivariant cohomology, following $[\overline{\mathrm{Cr}}],[\mathrm{Lu}],[\mathrm{Z}]$. After defining $\mathcal{B}$ and $\mathcal{F}_{[\omega]}$ in Section 4 they are reinterpreted in Section 5 as integrals of closed (holomorphically) equivariant forms, which are built out of equivariant extensions of the Kähler and Ricci forms. A by-product of these formulas is a simple derivation of a localization formula for the invariants, already found in $[\mathrm{T}]$ for the Futaki invariant. The invariants $\mathcal{B}$ and $\mathcal{F}_{[\omega]}$ are then placed in a family of $n+1$ invariants, each of which is related via the duality pairings to the elementary symmetric functions of the Ricci endomorphism. Finally, the two above-mentioned bilinear forms are described in holomorphic equivariant terms.

Sections 6 and 7 deal mainly with central metrics of the second type. Following a similar result in the extremal case [H2], a lower bound is derived on the $L^{2}$ norm of the Laplacian of the central curvature, for metrics in a fixed Kähler class, where the class satisfies a technical assumption. This topological bound is achieved exactly by central metrics of type II, and for these it is nonzero precisely when the central vector field is non-trivial. In Section $\mathbf{7}$ the equivalence of $\mathcal{B}$ and $\mathcal{F}_{c_{1}}$ is used to show that there exist manifolds for which at least a very closely related topological quantity is nonzero. This is significant since, unlike the case of metrics of the first type we do not give explicit existence results for type II central metrics with a non-trivial vector field. The section concludes by further applying the equivalence of the invariants on manifolds of Fano type to deduce relations between the central and extremal vector fields.

Section 8 gives existence and uniqueness results, almost exclusively for metrics of constant central curvature. The first striking fact is that Yau's solution to the Calabi conjecture implies that such metrics exist in any Kähler class, provided they exist in one such class. Also, unlike the situation for extremal metrics, the various 
central curvatures of central metrics on a given Kähler manifold are either all, or all not, constants. More importantly, because such metrics have Ricci forms of constant signature, vanishing theorems of Demailly $[\mathrm{Dm}]$ and others allow us to deduce, from their existence, the growth rates for the dimensions of cohomology groups alluded to above. This leads to a class-uniqueness theorem for such metrics on manifolds admitting non-Ricci-flat Kähler-Einstein metrics, and also to a partial classification in complex dimension two, featuring examples in all Kodaira dimensions. These considerations of existence and uniqueness imply that on a manifold of Fano type, Kähler-Einstein metrics exist if and only if metrics of constant central curvature exist in all classes.

Finally, in Section 9 we state the existence result for central metrics of the first type, and compare it to known results about extremal metrics locally conformal to Einstein metrics on the same class of spaces $[\mathrm{Dr}]$, [HS2]. The proof is given in [HM].

\section{ACKNowledgments}

The notion of a central metric originated in my thesis, and this work is in part a summary, in part a continuation, of the former. I would like to thank my advisor, Claude LeBrun, for years of instruction, in direct form, by example and through demonstration. In particular, I thank him for suggesting the classification above for Kähler surfaces. I would also like to thank Yael Karshon and Maxim Braverman for introducing me to recent vanishing theorems in many enlightening conversations, and Marina Ville for directing me to the reference $[\mathrm{Kb} 3$. Finally, I wish to thank Christina Wiis Tønnesen-Friedman for many clarifications regarding explicit constructions of extremal metrics, and Andrew Hwang for the successful collaboration in $[\mathrm{HM}]$.

\section{Ricci Determinant and Ricci Trace}

Let $\left(M^{n}, g\right)$ be a compact Kähler manifold. We denote by $\omega$ its Kähler form, and by $\rho$ its Ricci form. Recall that the two are related by formula (1).

A holomorphic vector field $\Xi:=\Xi_{f}$ is called gradient if its (metric) dual $(0,1)$ form is $\bar{\partial}$-exact:

$$
\imath_{\Xi_{f}} \omega=\bar{\partial} f .
$$

We also write $\Xi_{f}:=(\bar{\partial} f)^{\#}$. The function $f:=f_{\Xi}$ is determined up to an additive constant, and is called a holomorphy potential.

More generally, the Hodge decomposition of the $(0,1)$-form $\alpha$ metrically dual to a holomoprhic vector field $\Xi$ is of the form

$$
\alpha=\alpha_{H}+\bar{\partial} f,
$$

with $\alpha_{H}$ harmonic and $f$ a complex-valued function (where the term involving $\bar{\partial}^{*}$ vanishes by the holomorphicity of $\Xi$ and the Kähler condition). The component $\bar{\partial} f$ will be called the gradient part of the vector field, and at times the latter may still be denoted $\Xi_{f}$.

The $\bar{\partial}$-Laplacian $\Delta_{\bar{\partial}}:=\bar{\partial}^{*} \bar{\partial}+\bar{\partial} \bar{\partial}^{*}$ will be denoted simply $\Delta$.

Definition 2.1. The central curvature of $g$ is the function $C$ satisfying

$$
\rho^{\wedge n}=C \omega^{\wedge n} .
$$


A Kähler metric $g$ is called central of type I (II) if and only if $C(\Delta C)$ is a holomorphy potential, i.e., the vector field $\Xi_{C}:=(\bar{\partial} C)^{\#}\left(\Xi_{\Delta C}:=(\bar{\partial} \Delta C)^{\#}\right)$ is holomorphic. We call $\Xi_{C}\left(\Xi_{\Delta C}\right)$ the type I (II) central vector field.

Remark 2.2. The central curvature $C$ can also be characterized as the determinant of the Ricci endomorphism, or via the equation

$$
C:=\frac{\operatorname{det} \rho}{\operatorname{det} \omega} .
$$

Here det refers to the complex determinant, and $C$ is of course well defined because $\omega$ is non-degenerate. Note that if $C$ is constant, it is central of both types, and the corresponding vector field is the trivial one. Kähler-Einstein metrics (i.e., metrics for which $\rho=\lambda \omega$, with $\lambda$ constant) provide examples of central metrics with constant central curvature. When we refer to central metrics without mention of type, we always mean type I.

Remark 2.3. In Section 8 it will be shown that the existence of representative metrics of constant central curvature throughout the Kähler cone in $H^{1,1}(M)$, where $M$ is of Fano type, is guaranteed by such existence in the first Chern class $c_{1}$, where the metrics are necessarily Kähler-Einstein. This relation to distinguished elements in the central class of the cone is what inspired the above terminology. The volume forms of such metrics were called volume-Einstein in [Mb].

As mentioned in the introduction, in more than one occasion we will be comparing the central curvature with the scalar curvature, which is the metric trace of $\rho$,

$$
s=(\rho, \omega) .
$$

The equation for top-dimensional forms corresponding to (3) is

$$
s \omega^{\wedge n}=2 n \rho \wedge \omega^{\wedge n-1} .
$$

The associated distinguished metric notion was defined by Calabi in [C1]:

Definition 2.4. A Kähler metric $g$ is called extremal if it is a critical point of the functional

$$
g \longrightarrow \int_{M} s_{g}^{2} \frac{\omega^{\wedge n}}{n !}
$$

with domain the Kähler metrics having representatives in the class $[\omega]$. Here $s_{g}$ denotes the scalar curvature of $g$.

Over compact manifolds, this takes a form closer to that of the definition of a central metric, since one has

Proposition 2.5 (Calabi [C1]). For $M$ compact, a Kähler metric is extremal if and only if its scalar curvature is a holomorphy potential, i.e., $\Xi_{s}=(\bar{\partial} s)^{\#}$ is holomorphic. We call $\Xi_{s}$ the extremal vector field.

Here metrics of constant scalar curvature are associated with the trivial vector field, and again, Kähler-Einstein metrics serve as examples of the latter.

Remark 2.6. One may ask here what notion in the extremal setting corresponds to the division into types of central metrics. It seems that an investigation of Kähler metrics for which $\Delta s$ is a holomorphy potential has not been carried out. However, denoting by $\rho_{H}$ the harmonic component in the Hodge decomposition of 
the Ricci form, then, since $\rho$ and $\rho_{H}$ belong to the same Dolbeault class, Hodge theory guarantees that there exists a smooth real-valued function $F$, called the Ricci potential, such that

$$
\rho-\rho_{H}=i \partial \bar{\partial} F
$$

(cf. [GH, Chapter 1, Section 2]). The Ricci potential is determined only up to an additive constant. Kähler metrics for which it is a holomorphy potential are primary examples of Kähler-Ricci solitons, or quasi-Einstein metrics [ $\mathrm{Ki}], \mathrm{Co}],[\mathrm{Gu}]$, where $\bar{\partial} F$ is at least the gradient part of a holomorphic vector field. Now, taking traces in equation (77), we see that

$$
\Delta F=-\frac{1}{2}\left(s-s_{0}\right)
$$

where $s_{0}$ is the average scalar curvature, $s_{0}=\int_{M} s \frac{\omega^{\wedge n}}{n !} / \int_{M} \frac{\omega^{\wedge n}}{n !}$ (here the Hard Lefschetz theorem is used to conclude that the trace of $\rho_{H}$ is harmonic, and therefore constant).

Thus, Kähler-Ricci solitons together with extremal metrics may be considered the analogues of the two types of central metrics. Taking this point of view, one might associate $C$ with $F$ rather than $s$. To some degree this is supported by the cohomological treatment of the following sections. However, it is not a natural stance to take judging from the similarity of equations (3) and (5), or from the fact that both $s$ and $C$ are particular elementary symmetric functions of the Ricci endomorphism. It will be shown in Section 5 that the latter can all be treated essentially within the same framework.

\section{Holomorphic Equivariant Cohomology}

An invariant of a cohomological nature is associated to central metrics of either type. It is the analog of the well-known Futaki invariant. In this section we set up a proper cohomological background for the description of such invariants. Our treatment follows $[\mathrm{Lu}]$, see also $[\mathrm{Z}],[\mathrm{W},, \mathrm{Cr}],[\mathrm{Bt1}],[\mathrm{Bt2}]$.

For a compact complex manifold $\left(M_{n}, J\right)$, let

$$
\Omega^{(r)}(M)=\bigoplus_{q-p=r} \Omega^{p, q}(M)
$$

where $\Omega^{p, q}(M)$ denotes the smooth $(p, q)$-forms. Given a holomorphic vector field $\Xi$ on $M$, define the differential operator

$$
\bar{\partial} \Xi=\bar{\partial}-\imath \Xi .
$$

Since

$$
\bar{\partial}_{\Xi}^{2}=-\left(\bar{\partial} \imath_{\Xi}+\imath_{\Xi} \bar{\partial}\right)=0
$$

it follows that $\left(\Omega^{(*)}(M), \bar{\partial}_{\Xi}\right)$ constitutes a differential complex, where ${ }^{(*)}$ denotes the range $r=-n,-n+1, \ldots, n-1, n$. We denote the resulting cohomology by $H_{\Xi}^{(r)}(M)$, if $r$ is nonzero, and $H_{\Xi}(M)$ otherwise. Closed forms, or classes in the latter, admit a localization formula. To state it, we first describe the allowed degeneracy behavior of $\Xi$ at its zero locus.

Assume the vanishing locus $M_{0}$ of $\Xi$ is a (not necessarily connected) complex submanifold. Let $N=\left.T^{(1,0)} M\right|_{M_{0}} / T^{(1,0)} M_{0}$ be its holomorphic normal bundle, with locally constant complex rank denoted $\operatorname{rk}(N)$. 
Let $\mathcal{L}_{\Xi} \in \Gamma(\operatorname{End}(N))$ be the endomorphism of $N$ induced by the (complex) Lie derivative action on $\left.T^{(1,0)} M\right|_{M_{0}}$. We make the further assumption that $\mathcal{L}_{\Xi}$ is invertible. In this case we call $\Xi$ a non-degenerate vector field.

Integration of an equivariant form in $\Omega^{(0)}(M)$ against a submanifold of complex dimension $p$ is defined by integrating its component $(p, p)$-form. We have

Theorem 1 (Holomorphic Localization $[\mathrm{Lu})$. Let $M, M_{0}$ and $N$ be as above, with $\Xi$ non-degenerate. Given $\alpha$ with $[\alpha] \in H_{\Xi}(M)$,

$$
\int_{M} \alpha=(-2 \pi)^{\operatorname{rk}(N)} \int_{M_{0}} \frac{\alpha}{\operatorname{det}\left(\mathcal{L}_{\Xi}+\Omega\right)},
$$

where $\Omega$ is the (complex endomorphism-valued) curvature 2-form of an $\mathcal{L}_{\Xi}$-invariant connection on $N$, induced from any Hermitian metric on $N$, and the determinant is complex.

Remark 3.1. Returning to the case of a Kähler manifold with Kähler form $\omega$, if $\Xi_{f}$ is a gradient vector field with holomorphy potential $f$, then $\omega+f$ is a (holomorphic) equivariantly closed form, by equation (21). One can also consider equivariantly closed extensions of the Ricci form $\rho$. In fact, for a gradient vector field,

$$
\imath_{\Xi_{f}} \rho=\bar{\partial}(\Delta f)=\Delta\left(\imath_{\Xi_{f}} \omega\right),
$$

and this relation implies (on compact Kähler manifolds) that the vector field is holomorphic. In particular, it implies relation (2) (see [Bc2], $\mathrm{Yn}$, or Kb1, Theorem 4.2] for a statement involving any holomorphic vector field). Thus, a Kähler metric together with a gradient holomorphic vector field $\Xi_{f}$ induces two holomorphic equivariantly closed forms: $\omega+f$ and $\rho+\Delta f$.

For a general holomorphic vector field these forms are not closed. Note that if a holomorphic vector field $\Xi$ contains a non-trivial harmonic form in its Hodge decomposition, then it has an empty zero set ([Kb1, Corollary 4.5]). Such a vector field always gives rise to trivial holomorphic equivariant cohomology $[\mathrm{Lu}]$. So, with respect to it, all $\bar{\partial}_{\Xi}$-closed forms have vanishing integrals. In Section 5 we will have cause to consider even integrals constructed from the above non-closed forms.

Remark 3.2. We briefly mention the relation to symplectic geometry and the de Rham model for Borel equivariant cohomology. If the holomorphy potential $f$ of a gradient vector field is real, then the real part $X$ of $\Xi_{f}$ is a Killing vector field, and also a Hamiltonian vector field with respect to the Kähler form. When $\Xi$ generates a $\mathbb{C}^{*}$ action, its real part $X$ generates a circle action, and $f$ is the corresponding moment map. The forms $\omega+f$ and $\rho+(1 / 2) \Delta_{d} f$, with $\Delta_{d}$ the $d$-Laplacian, will now be equivariantly closed with respect to $d_{X}=d-\imath_{X}$. The corresponding localization formula for this operator is then equivalent to Theorem[1.

\section{Cohomological Metric Obstruction Invariants}

In the next section we will associate to each Kähler class in $H^{1,1}(M)$ a family of invariants. Each of them gives information about Kähler metrics in the class with a distinguished curvature potential function. These potentials are essentially the elementary symmetric functions of the Ricci endomorphism. The non-vanishing of one of these invariants obstructs the existence of metrics having a corresponding constant curvature potential function. 
Presently, we focus on the invariants corresponding to the trace and the determinant, i.e., the scalar and central curvatures. The former is the Futaki invariant, and we begin by reviewing its most basic features.

Definition 4.1. Let $\left(M_{n}, \omega\right)$ be a compact Kähler manifold with Ricci potential $F$. The Futaki character is the map $\mathcal{F}_{[\omega]}: h(M) \rightarrow \mathbb{C}$, where $h(M)$ denotes the Lie algebra of holomorphic vector fields on $M$, given by

$$
\mathcal{F}_{[\omega]}(\Xi)=\int_{M} \Xi F \frac{\omega^{\wedge n}}{n !} .
$$

Remark 4.2. That existence of constant scalar curvature metrics in $[\omega]$ implies $\mathcal{F}_{[\omega]} \equiv 0$ follows from equation $(8)$, since it shows that $s$ is constant if and only if $F$ is constant.

Remark 4.3. The values of this functional do not depend on the choice of metric in the Kähler class $[\omega]$, i.e., it is a Kähler class invariant [B], [C2], [Ft3]. We will soon indicate how this follows from holomorphic equivariant considerations. Note also that the invariance implies that $\mathcal{F}_{[\omega]}$ is a Lie algebra character (cf. [C2]).

Remark 4.4. The value the Futaki invariant takes on a given holomorphic vector field is determined only by its gradient part (cf. T]). Recalling the Hodge decomposition $\alpha=\alpha_{H}+\bar{\partial} f$ of the $(0,1)$-form metrically dual to such a vector field, we see that the Futaki invariant evaluates to

$$
\mathcal{F}_{[\omega]}(\Xi)=\int_{M} \Xi F \frac{\omega^{\wedge n}}{n !}=\int_{M}\left(\alpha_{H}, \bar{\partial} F\right) \frac{\omega^{\wedge n}}{n !}+\int_{M}(\bar{\partial} f, \bar{\partial} F) \frac{\omega^{\wedge n}}{n !},
$$

where $(\cdot, \cdot)$ denotes the pointwise inner product induced on $(0,1)$-forms by the Kähler metric. But the first term on the right-hand side vanishes after integration by parts, since $\alpha_{H}$ is co-closed. In considering the value of $\mathcal{F}_{[\omega]}$, it is thus allowable to ignore the harmonic part of the vector field.

We now turn to the invariant corresponding to the determinant of the Ricci endomorphism, i.e., the central curvature. Denoting it by $\mathcal{B}$, it is defined as

$$
\mathcal{B}(\Xi):=\int_{M} \operatorname{div}(\Xi) \frac{\rho^{\wedge n}}{n !},
$$

with div denoting the complex divergence. Using equation (3) and an integration by parts, one sees that indeed $\mathcal{B} \equiv 0$ when $C$ is constant. Once again, $\mathcal{B}$ is also completely determined by its values on the gradient parts of the holomorphic vector fields. One difference from the Futaki invariant is that $\mathcal{B}$ does not depend even on the choice of Kähler class, but only on the complex structure of $M$, and can actually be defined more generally for Hermitian manifolds if $\rho$ is taken to be the first Chern form [FR1], FR2]. Thus, the non-vanishing of $\mathcal{B}$ obstructs the existence of any metric with constant central curvature on a given complex manifold.

\section{Holomorphic Equivariant Interpretation of the InVARIANts}

Both the Futaki invariant and $\mathcal{B}$ may be interpreted in more than one cohomological framework (cf. [B], [Ft2], FT], [Ts1], [Ts2], [Yo]). Here we show that both invariants discussed in the previous section, and others that we will define, can be understood as integrals of closed equivariant forms built out of the two basic ones, $\omega+f$ and $\rho+\Delta f$, associated to the gradient vector field $\Xi_{f}$. Since we have seen that the values of these invariants depend only on the gradient part of a given 
holomorphic vector field, the formulas obtained below will hold generally for any holomorphic vector field, even though the above equivariant forms are no longer $\bar{\partial}_{\Xi}$-closed. In the non-gradient case they are, at least in some cases, vanishing formulas. For example, if a vector field is parallel with respect to some metric on the manifold, all Futaki invariants vanish on it [LSm], and so even if its gradient part with respect to another metric does not vanish, the associated integrals still evaluate to zero.

Proposition 5.1. Let $\left(M_{n}, g\right)$ be a compact Kähler manifold, and $\Xi_{f}$ a holomorphic vector field with gradient part $\bar{\partial} f$. One then has:

$$
\begin{aligned}
\mathcal{F}_{[\omega]}\left(\Xi_{f}\right) & =\frac{1}{2(n+1) !} s_{0} \int_{M}(\omega+f)^{\wedge n+1}-\frac{1}{n !} \int_{M}(\rho+\Delta f) \wedge(\omega+f)^{\wedge n}, \\
\mathcal{B}\left(\Xi_{f}\right) & =-\frac{1}{(n+1) !} \int_{M}(\rho+\Delta f)^{\wedge n+1} .
\end{aligned}
$$

Proof. For such a vector field, we have

$$
\begin{aligned}
\mathcal{F}_{[\omega]}\left(\Xi_{f}\right) & =\int_{M} \Xi_{f} F \frac{\omega^{\wedge n}}{n !}=\int_{M}(\partial F, \partial f) \frac{\omega^{\wedge n}}{n !}=\int_{M} \Delta_{\partial} F f \frac{\omega^{\wedge n}}{n !} \\
& =\int_{M} f \Delta F \frac{\omega^{\wedge n}}{n !}=-\frac{1}{2} \int_{M} f\left(s-s_{0}\right) \frac{\omega^{\wedge n}}{n !}
\end{aligned}
$$

where we have used the equality of the $\bar{\partial}$-Laplacian $\Delta$ with the $\partial$-Laplacian $\Delta_{\partial}$. The last equality in (13) follows from relation (8).

By relation (5), the Futaki invariant equals

$$
\frac{1}{2 n !} s_{0} \int_{M} f \omega^{\wedge n}-\frac{1}{(n-1) !} \int_{M} f \rho \wedge \omega^{\wedge n-1} .
$$

The right-hand side of expression (12), on the other hand, evaluates to

$$
\begin{gathered}
\frac{1}{2(n+1) !} s_{0}(n+1) \int_{M} f \omega^{\wedge n}-\frac{1}{n !} \int_{M} \rho \wedge\left(\sum_{k=0}^{n}\left(\begin{array}{l}
n \\
k
\end{array}\right) f^{n-k} \omega^{\wedge k}\right) \\
-\frac{1}{n !} \int_{M} \Delta f\left(\sum_{k=0}^{n}\left(\begin{array}{l}
n \\
k
\end{array}\right) f^{n-k} \omega^{\wedge k}\right)=\frac{1}{2 n !} s_{0} \int_{M} f \omega^{\wedge n}-\frac{1}{n !} n \int_{M} f \rho \wedge \omega^{\wedge n-1} \\
-\frac{1}{n !} \int_{M} \Delta f \omega^{\wedge n}=\frac{1}{2 n !} s_{0} \int_{M} f \omega^{\wedge n}-\frac{1}{(n-1) !} \int_{M} f \rho \wedge \omega^{\wedge n-1},
\end{gathered}
$$

where we have used the Divergence Theorem in the last step. Comparing with (14), we are done. The computation for $\mathcal{B}$ is immediate.

Combining this proposition with the Holomorphic Localization Theorem completes a rather simple derivation of the localization formula for the two invariants. For the Futaki invariant, an equivalent formula was already given in $[\mathrm{T}]$ :

Theorem 2. Let $\left(M_{n}, J\right)$ be a Kähler manifold, $\Xi$ a non-degenerate holomorphic vector field and $\omega$ a Kähler form of an arbitrary Kähler metric $g$ on $M$. Denote by $\rho$ the Ricci form of $g$, and by $\bar{\partial} f$ the gradient part of $\Xi$ with respect to the metric. 
Then

$$
\begin{aligned}
& \mathcal{F}_{[\omega]}\left(\Xi_{f}\right)=(-2 \pi)^{\mathrm{rk}(N)}\left\{\frac{1}{2(n+1) !} s_{0} \int_{M_{0}} \frac{(\omega+f)^{\wedge n+1}}{\operatorname{det}\left(\mathcal{L}_{\Xi}+\Omega\right)}\right.\left.-\frac{1}{n !} \int_{M_{0}} \frac{(\rho+\Delta f) \wedge(\omega+f)^{\wedge n}}{\operatorname{det}\left(\mathcal{L}_{\Xi}+\Omega\right)}\right\}, \\
& \mathcal{B}\left(\Xi_{f}\right)=(-2 \pi)^{\mathrm{rk}(N)}\left\{-\frac{1}{(n+1) !} \int_{M_{0}} \frac{(\rho+\Delta f)^{\wedge n+1}}{\operatorname{det}\left(\mathcal{L}_{\Xi}+\Omega\right)}\right\},
\end{aligned}
$$

where $M_{0}$, rk, det, $\mathcal{L}_{\Xi}, \Omega$ are as in Theorem 1 .

For a gradient vector field $\Xi_{f}$ generating a $\mathbb{C}^{*}$ action, this localization theorem was used to obtain explicit formulas in dimension 4 for $\mathcal{F}_{[\omega]}$ in $\underline{\mathrm{Ms}}$, generalizing those of [LSm]. The Morse-theoretic properties of the moment map $f$ were employed to this end (see Remark 3.2).

Our main interest lies, however, in formula (12) itself. Assume, for simplicity only, that $\Xi$ is a gradient vector field. Since the holomorphy potential $f$ is only determined up to an additive constant, normalizing $f$ by setting

$$
\frac{1}{(n+1) !} \int_{M}(\omega+f)^{\wedge n+1}=\int_{M} f \frac{\omega^{\wedge n}}{n !}=0
$$

gives the simplified formula

$$
\mathcal{F}_{[\omega]}\left(\Xi_{f}\right)=-\frac{1}{n !} \int_{M}(\rho+\Delta f) \wedge(\omega+f)^{\wedge n} .
$$

Comparing with the expression for $\mathcal{B}\left(\Xi_{f}\right)$, one is led to consider other invariants linear in $\Xi_{f}$. Consider the family of $n+1$ expressions

$$
\mathcal{A}_{k}\left(\Xi_{f}\right)=\int_{M}(\rho+\Delta f)^{\wedge k} \wedge(\omega+f)^{\wedge n+1-k}, \quad k=1, \ldots, n+1,
$$

with $-n ! \mathcal{F}_{[\omega]}=\mathcal{A}_{1}$ and $-(n+1) ! \mathcal{B}=\mathcal{A}_{n+1}$. All the $\mathcal{A}_{k}$ 's are Kähler class invariants: a change of Kähler representative $\omega \rightarrow \omega+i \partial \bar{\partial} \phi$ results in a change $\omega+f \rightarrow$ $\omega+f+\bar{\partial}_{\Xi_{f}}(-i(\partial \phi))+K$, where $K$ is some constant. The latter may be ignored as in the normalization (15) (otherwise one can change the definition of the invariants by subtracting a multiple of $c_{1}^{\wedge k} \cup[\omega]^{\wedge n-k} \int_{M}(\omega+f)^{\wedge n+1}$, similar to the $s_{0}$ term in the expression for the Futaki invariant in Proposition 5.1). Similarly, if the Ricci form transforms as $\rho \rightarrow \rho+\bar{\partial} \beta$, for a $(1,0)$-form $\beta$, then $\rho+\Delta f \rightarrow \rho+\Delta f+\bar{\partial}_{\Xi_{f}} \beta$ (here there is no extra constant, since the value of the Laplacian at the maximum of $f$ has an invariant meaning, which can be seen from the localization formula, see [T]). Thus both forms change only by an equivariantly exact form, giving the invariance.

Other than $\mathcal{B}$ and $\mathcal{F}_{[\omega]}$, we will also be employing two bilinear forms on gradient holomorphic vector fields. These are

and

$$
\mathcal{K}_{[\omega]}^{I}\left(\Xi_{1}, \Xi_{2}\right)=\int_{M} f_{\Xi_{1}} \Delta f_{\Xi_{2}} \frac{\omega^{\wedge n}}{n !}
$$

$$
\mathcal{K}_{[\omega]}^{I I}\left(\Xi_{1}, \Xi_{2}\right)=\int_{M} f_{\Xi_{1}} f_{\Xi_{2}} \frac{\omega^{\wedge n}}{n !}
$$

where for the bilinearity of $\mathcal{K}_{[\omega]}^{I I}$ one assumes explicitly that the holomorphy potentials $f_{\Xi_{i}}$ are normalized as in (15). 
The Kähler class invariance of $\mathcal{K}_{[\omega]}^{I I}$ was proved in [FM]. It can be understood via equivariant cohomology, since $\mathcal{K}_{[\omega]}^{I I}$ can be written as an integral of equivariantly closed forms:

$\mathcal{K}_{[\omega]}^{I I}\left(\Xi_{1}, \Xi_{2}\right)=\frac{\left\{\int_{M}\left(\omega+f_{\Xi_{1}}\right) \wedge\left(\omega+f_{\Xi_{2}}\right)^{\wedge n+1}-\left(\left(\begin{array}{c}n+1 \\ 2\end{array}\right) /\left(\begin{array}{c}n+2 \\ 2\end{array}\right)\right) \int_{M}\left(\omega+f_{\Xi_{2}}\right)^{\wedge n+2}\right\}}{\left(\begin{array}{c}n+1 \\ 1\end{array}\right)}$.

The invariance of $\mathcal{K}_{[\omega]}^{I}$ can also be seen via equivariant cohomology, because $\Xi_{1}+\Xi_{2}$ has holomorphy potential $f_{1}+f_{2}$, and

$$
\begin{aligned}
\mathcal{K}_{[\omega]}^{I}\left(\Xi_{1}, \Xi_{2}\right)=\frac{1}{n+1}\{ & \int_{M}\left(\omega+f_{1}\right)^{\wedge n+1}\left(\rho+\Delta f_{1}+\Delta f_{2}\right) \\
& \left.-\int_{M}\left(\omega+f_{1}\right)^{\wedge n+1}\left(\rho+\Delta f_{1}\right)\right\} .
\end{aligned}
$$

Remark 5.2. In conclusion, note that at least within the framework presented here, for both bilinear forms the assumption that they act only on gradient vector fields is now necessary, and one no longer has available the argument allowing the consideration of gradient parts of other vector fields. However, there is at least complete freedom in the choice of metric, since if a vector field has zeros, it is gradient with respect to any Kähler metric ([Kb1, Corollary 4.5]), and any gradient vector field has zeros [Lc2] [Lc2], [LSm].

\section{Lower Bounds on $L^{2}$ Curvature Quantities}

We have seen that $\mathcal{B}$ provides an obstruction to the existence of metrics of constant central curvature. In this section its relation to arbitrary type II central metrics is examined. Again the situation is compared with that of the scalar curvature. For extremal Kähler metrics, the basic relation between them and the Futaki invariant is that the latter is, up to an unimportant factor, $\mathcal{K}_{[\omega]}^{I I}$-dual to the extremal vector field $\Xi_{s}=\Xi_{s-s_{0}}[\mathrm{FM}]$ :

$$
-2 \mathcal{F}_{[\omega]}\left(\Xi_{f}\right)=\int_{M} f_{\Xi}\left(s-s_{0}\right) \frac{\omega^{\wedge n}}{n !}=\mathcal{K}_{[\omega]}^{I I}\left(\Xi_{f}, \Xi_{s-s_{0}}\right)
$$

by relation (13). More precisely, this duality holds perfectly on the reductive quotient algebra of the Lie subalgebra of gradient vector fields, where $\mathcal{K}_{[\omega]}^{I I}$ induces a non-degenerate pairing. (Similarly, $\mathcal{F}_{[\omega]}$ is $\mathcal{K}_{[\omega]}^{I}$-dual to the distinguished vector field of the Kähler-Ricci solitons mentioned in Section 2.) Recall here that the Albanese map of $M$ induces a Lie group homomorphism between the group of holomorphic automorphisms of $M$ and that of the Albanese torus. Its kernel is a linear algebraic group whose Lie algebra coincides with the Lie subalgebra of gradient vector fields [Lc2], [LSm], [FM].

Remark 6.1. Of all the linear invariants $\mathcal{A}_{k}$, the first and last ones, $-n ! \mathcal{F}_{[\omega]}=\mathcal{A}_{1}$ and $-(n+1) ! \mathcal{B}=\mathcal{A}_{n+1}$, may be considered the simplest, in the sense that in the duality schemes we employ they are the only ones that determine curvature potential functions that are given by a single elementary symmetric function. The typical invariant $\mathcal{A}_{k}$ is $\mathcal{K}_{[\omega]}^{I I}$-dual to a gradient vector field whose holomorphy potential is a linear combination of an elementary symmetric function and the Laplacian of an adjacent one in the characteristic polynomial of the Ricci endomorphism. 
Returning to the extremal case, the above duality implies of course that if the extremal vector field is non-trivial, the Futaki invariant evaluates non-trivially on it. We now review an $L^{2}$ lower bound that follows from this duality and holds for all metrics in a fixed Kähler class $[\mathrm{FM}]$.

For $(M, g)$ Kähler, denote the $L^{2}$ inner product on smooth functions by

$$
\langle f, h\rangle:=\int_{M} f \bar{h} \frac{\omega^{\wedge n}}{n !}
$$

with corresponding norm denoted $\|\cdot\|_{g}$.

Let $C_{0}^{\infty}(M, \mathbb{C}, g)$ be the space of all smooth complex-valued functions on $M$ that are $\langle.,$.$\rangle -perpendicular to the constants.$

Let $\Gamma_{0}:=\Gamma_{0, g}$ be its (finite-dimensional) subspace of all $g$-holomorphy potentials also $\langle.,$.$\rangle -perpendicular to the constants.$

Let $\pi_{g}: C_{0}^{\infty}(M, \mathbb{C}, g) \rightarrow \Gamma_{0}$ be the orthogonal projection with respect to $\langle.,$.$\rangle .$

Theorem 3 (Futaki-Mabuchi [FM], [H2]). Let $(M,[\omega])$ be a polarized compact Kähler manifold, for which the kernel of $\mathcal{K}_{[\omega]}^{I I}$ is included in the derived subalgebra of the gradient vector fields. If $g$ is any Kähler metric with Kähler form representing a Kähler class $[\omega]$, and with scalar curvature $s$ and average scalar curvature $s_{0}$, then the number $\mathcal{F}_{[\omega]}\left(\Xi_{\pi_{g}\left(s-s_{0}\right)}\right)$ depends only on the Kähler class.

$\Xi_{\pi_{g}\left(s-s_{0}\right)}$ will still be called the extremal vector field of $g$.

This was combined in $\underline{\mathrm{H} 2}$ with

Theorem 4 (Hwang [H1], [H2], see also [Sm]). Let $(M,[\omega])$ be a compact Kähler manifold. With notations (but not the further Lie algebraic assumption) as in Theorem 3 ,

$$
\int_{M}\left(s-s_{0}\right)^{2} \frac{\omega^{\wedge n}}{n !} \geq-2 \mathcal{F}_{[\omega]}\left(\Xi_{\pi_{g}\left(s-s_{0}\right)}\right) .
$$

The right-hand side of this inequality is real and nonnegative, and equality occurs exactly when $g$ is extremal.

Remark 6.2. Since for polarized manifolds $(M,[\omega])$ as in Theorem 3 , the real number on the right-hand side of inequality (19) depends only on the Kähler class [ $\omega]$, it gives, in combination with Theorem 4 , a lower bound for the left-hand side, over all Kähler metrics in the class.

Focusing back on central metrics, type I (II) central vector fields are $\mathcal{K}_{[\omega]^{I}}^{I}$-dual $\left(\mathcal{K}_{[\omega]}^{I I}\right.$-dual $)$ to $\mathcal{B}$ in the same sense as above. So again, $\mathcal{B}$ evaluates non-trivially on these vector fields (whenever they themselves are non-trivial). To save space, we give the analog of the above $L^{2}$ lower bound only for type II central vector fields, for which the needed properties of $\mathcal{K}_{[\omega]}^{I I}$ have already been checked in [FM].

Theorem 5. Let $(M,[\omega])$ be a compact polarized Kähler manifold satisfying the assumption of Theorem [. For any Kähler metric $g$ with Kähler form representing $[\omega]$, the number $\mathcal{B}\left(\Xi_{\pi_{g}(\Delta C)}\right)$ depends only on the Kähler class.

Again the definition of the (type II) central vector field of a metric $g$ is now extended to $\Xi_{\pi_{g}(\Delta C)}$. 
Theorem 6. Let $M$ be a compact Kähler manifold. Let g be a Kähler metric on $M$ with Kähler form $\omega$ and central curvature $C$. Then

$$
\int_{M}(\Delta C)^{2} \frac{\omega^{\wedge n}}{n !} \geq-\mathcal{B}\left(\Xi_{\pi_{g}(\Delta C)}\right),
$$

where $\pi_{g}: C_{0}^{\infty}(M, \mathbb{C}, g) \rightarrow \Gamma_{0}$ is as in Theorem 4. The right-hand side of inequality (20) is real and nonnegative, with equality occuring exactly when $g$ is central of type II.

The proofs of the above results are very similar to those of the first set of statements. We review the most important steps. The pertinent Lie-theoretic background here is as follows. The Lie algebra of gradient vector fields was described above to be the Lie algebra of a linear algebraic group. Corresponding to the semidirect splitting of this group, the Lie algebra decomposes as a sum of a nilpotent radical and a reductive quotient algebra $[\mathrm{Fj}]$. The latter's embedding and, consequently, the splitting, are non-canonical. However, if a metric has a group of isometries maximally compact in this linear algebraic group, the reductive algebra is realized as a subalgebra of the gradient vector fields by complexifying the Lie algebra of the corresponding non-parallel Killing fields. Note that by a standard averaging argument, in any fixed Kähler class there is always a metric satisfying the maximal compactness property. It was proven in [FM] that for any two such metrics, the corresponding extremal vector fields (in the extended sense) belong to the centers of the corresponding reductive realizations, and furthermore, one such vector field is mapped to the other by the differential of the adjoint action of an element of the unipotent radical. See the relevant papers quoted in this section for further information.

Proof of Theorem 5 . Let $\rho$ be the Ricci form of $g$, and note that $C$ is real-valued and satisfies $\rho^{\wedge n}=C \omega^{\wedge n}$. We now have, for any vector field $\Xi_{f}$,

$$
\begin{aligned}
\mathcal{B}\left(\Xi_{f}\right) & =\int_{M}\left(\Delta f_{\Xi}\right) \frac{\rho^{\wedge n}}{n !}=\int_{M}\left(\Delta f_{\Xi}\right) C \frac{\omega^{\wedge n}}{n !}=\int_{M} f_{\Xi} \Delta C \frac{\omega^{\wedge n}}{n !} \\
& =\int_{M} f_{\Xi} \pi_{g}(\Delta C) \frac{\omega^{\wedge n}}{n !}=\mathcal{K}_{[\omega]}^{I I}\left(\Xi_{f}, \Xi_{\pi_{g}(\Delta C)}\right),
\end{aligned}
$$

where we have used integration by parts, and then, in the penultimate step, the orthogonality of the projection $\pi_{g}$.

Therefore, because for any $g$ with Kähler form belonging to $[\omega]$, the vector field $\Xi_{\pi_{g}(\Delta C)}$ is $\mathcal{K}_{[\omega]}^{I I}$-dual to the fixed invariant functional $\mathcal{B}$, as $g$ varies among metrics with Kähler form in $[\omega]$, this vector field can only change by an addition of a vector in the subspace of the Lie algebra of vector fields on which $\mathcal{K}_{[\omega]}^{I I}$ degenerates. Since $\mathcal{K}_{[\omega]}^{I I}$ is metric independent, so is its degeneracy subspace. But $\mathcal{B}$, being a character, vanishes on vectors of this subspace by the assumption on the Lie algebra. Thus, although $\Xi_{\pi_{g}(\Delta C)}$ may vary with the metric, $\mathcal{B}\left(\Xi_{\pi_{g}(\Delta C)}\right)$ does not, and we are done.

Proof of Theorem [6. We write the $L_{g}^{2}$-orthogonal decomposition of $\Delta C$ as

$$
\Delta C=\pi_{g}(\Delta C)+T \text {. }
$$


Then,

$$
\begin{aligned}
0 & \leq\left\langle\pi_{g}(\Delta C), \pi_{g}(\Delta C)\right\rangle=\left\langle\pi_{g}(\Delta C), \Delta C-T\right\rangle \\
& =\left\langle\pi_{g}(\Delta C), \Delta C\right\rangle=\left\langle\Delta\left(\pi_{g}(\Delta C)\right), C\right\rangle \\
& =-\int_{M} \operatorname{div}_{g} \Xi_{\pi_{g}(\Delta C)} C \frac{\omega^{\wedge n}}{n !}=-\int_{M} \operatorname{div}_{g} \Xi_{\pi_{g}(\Delta C)} \frac{\rho^{\wedge n}}{n !}=-\mathcal{B}\left(\Xi_{\pi_{g}(\Delta C)}\right),
\end{aligned}
$$

where first we have used the orthogonality of the relation (22), then the fact that $\Delta C$ is real-valued implied that conjugation was unnecessary in the integral, and finally integration by parts. Combining this again with the orthogonality of condition (22), we have

$$
\begin{aligned}
\|\Delta C\|_{g}^{2} & =\left\|\pi_{g}(\Delta C)\right\|_{g}^{2}+\|T\|_{g}^{2} \\
& =-\mathcal{B}\left(\Xi_{\pi_{g}(\Delta C)}\right)+\|T\|_{g}^{2} \geq-\mathcal{B}\left(\Xi_{\pi_{g}(\Delta C)}\right),
\end{aligned}
$$

as required. Equality here exactly means $T=0$, or $\Delta C=\pi_{g}(\Delta C)$; so $g$ is central.

\section{Extremal and Type II Central Vector Fields}

In this section we employ known results about extremal metrics to show that non-trivial type II central vector fields do, in fact, exist, and to relate them to extremal vector fields. To this end, we first recall the relationship between the Futaki invariant and $\mathcal{B}$.

Proposition 7.1 (Futaki-Morita [FR2]). Let $M$ be a compact Fano manifold. Let $g$ be a Kähler metric with Kähler form $\omega$ representing the Kähler class $c_{1}$. If $F$ denotes the Ricci potential of $g$, then for any holomorphic vector field $\Xi$,

$$
\mathcal{F}_{c_{1}}(\Xi)=\int_{M} \Xi(F) \frac{\omega^{\wedge n}}{n !}=\int_{M} \operatorname{div}_{g} \Xi \frac{\rho^{\wedge n}}{n !}=\mathcal{B}(\Xi) .
$$

This was originally proved using the Calabi-Yau theorem. From the point of view of holomorphic equivariant cohomology, the proposition follows since both sides of equation (23), which are equal to integrals of closed equivariant forms, are also equal to the integral of another equivariantly closed form, namely (a multiple of) $\left(\rho_{H}+\Delta_{F} f\right)^{\wedge n+1}$, with $\Delta_{F} f:=\Delta f-(\partial F, \partial f)$ (see [Ft2]). Put in another, and simpler way, both forms belong to the same equivariant class.

In the following theorem only, the definition of $\pi_{g}$ is slightly altered.

Theorem 7. After a possible reinterpretation of $\pi_{g}$, there exist polarized manifolds with Kähler metrics satisfying

$$
-\mathcal{B}\left(\Xi_{\pi_{g}(\Delta C)}\right)>0
$$

and for which this number is a Kähler class invariant.

Proof. Let $M$ be a Fano manifold having an extremal Kähler metric $g$ of nonconstant scalar curvature in the class $c_{1}$. An example of such a manifold would be the one-point blow-up of $\mathbb{C P}^{2}$ (cf. [C1]). Such a manifold either satisfies the assumptions of Theorems 3 and 5 (cf. $\underline{\mathrm{Mb}}$ ), or in any case the metric satisfies the maximal compactness property [C2]. By the equality of functionals in Proposition 7.1, $\mathcal{B}$ is $\mathcal{K}_{c_{1}}^{I I}$-dual to both $-(1 / 2) \Xi_{s-s_{0}}$ and $\Xi_{\pi_{g}(\Delta C)}$, at least when the projection $\pi_{g}$ is taken to be the one in [FM], mapping onto holomorphy potentials corresponding to 
the reductive quotient algebra. Therefore these must differ by an element on which the character $\mathcal{B}$ vanishes; hence it takes the same value on both vector fields. Thus

$$
-\mathcal{B}\left(\Xi_{\pi_{g}(\Delta C)}\right)=-\mathcal{F}_{c_{1}}\left(-\frac{1}{2} \Xi_{s-s_{0}}\right)>0,
$$

where the strict inequality holds since $\Xi_{s-s_{0}}$ is non-trivial.

Combined with Theorem 6 this provides evidence for the possible existence of type II central metrics of non-constant central curvature. Note also that the above implies that $\pi_{g}$ projects $\Delta C$ onto the sum of $-(1 / 2)\left(s-s_{0}\right)$ and a holomorphy potential of a vector field in the degeneracy kernel of $\mathcal{K}_{c_{1}}^{I I}$.

The following is also a useful observation on the relation between these two vector fields:

Lemma 7.2. The following relation holds between the extremal and the type II central vector fields of any Kähler metric $g$ with Kähler form $\omega$ :

$$
\mathcal{F}_{[\omega]}\left(\Xi_{\pi_{g}(\Delta C)}\right)=\mathcal{B}\left(-\frac{1}{2} \Xi_{\pi_{g}\left(s-s_{0}\right)}\right) .
$$

Proof. We use orthogonality, reality, and integration by parts, as before:

$$
\begin{aligned}
\mathcal{F}_{[\omega]} & \left(\Xi_{\pi_{g}(\Delta C)}\right) \\
& =-\frac{1}{2} \int_{M} \pi_{g}(\Delta C)\left(s-s_{0}\right) \frac{\omega^{\wedge n}}{n !}=-\frac{1}{2} \int_{M} \pi_{g}(\Delta C) \pi_{g}\left(s-s_{0}\right) \frac{\omega^{\wedge n}}{n !} \\
& =-\frac{1}{2} \int_{M} \pi_{g}\left(s-s_{0}\right) \Delta C \frac{\omega^{\wedge n}}{n !}=-\frac{1}{2} \int_{M} \Delta\left(\pi_{g}\left(s-s_{0}\right)\right) C \frac{\omega^{\wedge n}}{n !} \\
& =-\frac{1}{2} \int_{M} \Delta\left(\pi_{g}\left(s-s_{0}\right)\right) \frac{\rho^{\wedge n}}{n !}=\mathcal{B}\left(-\frac{1}{2} \Xi_{\pi_{g}\left(s-s_{0}\right)}\right) .
\end{aligned}
$$

Combining this with Proposition [7.1 we get:

Corollary 7.3. For any Kähler metric with Kähler form $\omega$ on a manifold $M$ of Fano type,

$$
\mathcal{F}_{[\omega]}\left(\Xi_{\pi_{g}(\Delta C)}\right)=\mathcal{F}_{c_{1}}\left(-\frac{1}{2} \Xi_{\pi_{g}\left(s-s_{0}\right)}\right) .
$$

Note that that it is immaterial here that $g$ may not have, in general, a Kähler form representing $c_{1}$, since Proposition 7.1 is an equality of functionals, which in particular will be equal on the (metric independent) holomorphic vector field $-(1 / 2) \Xi_{\pi_{g}\left(s-s_{0}\right)}$.

Furthermore, it follows from this relation that for a type II central metric in $c_{1}$ with a non-trivial central vector field, the $L^{2}$ projection onto the line generated by this vector field (which is generally different from $\pi_{g}$ ) takes $-(1 / 2) \Xi_{s-s_{0}}$ onto $\Xi_{\Delta C}$ (here we identified gradient potentials with vector fields). A similar conclusion follows for extremal metrics.

\section{Existence and Uniqueness of Metrics with Constant Central Curvature}

As we have seen in previous sections, a large part of the framework for consideration of extremal Kähler metrics can be imitated for central Kähler metrics. 
However, with regards to existence and uniqueness, their behaviour appears nonidentical. We have already mentioned that central metrics of constant and nonconstant central curvature (of either type) cannot exist jointly on a given manifold. This is in contrast with the situation for extremal metrics, where such joint existence is possible, with the metrics belonging to different Kähler classes. A complete answer to the existence problem for extremal metrics is, however, not presently known. Furthermore, at least for a particular class of examples, existence throughout the Kähler cone and good uniqueness properties in a fixed Kähler class do not hold jointly [Tf]. This section describes the very uniform existence and uniqueness properties of central metrics of constant central curvature, under the assumption that one such metric is given.

Recall first the Calabi-Yau Theorem.

Theorem 8 (Calabi-Yau $\mathrm{Yu}$ ). Let $M$ be a compact Kähler manifold. If $\bar{\rho}$ is a real closed $(1,1)$-form representing $c_{1}(M)$ (if $\Psi$ is a real positive non-degenerate $(n, n)$-form), then in every Kähler class there exists a unique Kähler form $\omega$, whose Ricci form equals $\bar{\rho}$ (whose volume form equals a positive constant multiple of $\Psi$ ).

From this one derives the following consequence.

Theorem 9. If $M$ is a Kähler manifold admitting a central Kähler metric of constant central curvature, then every Kähler class in $H^{1,1}(M)$ is represented by (a Kähler form of) such a metric.

Proof. Let $g$ be a central metric with Kähler form $\omega$, Ricci form $\rho$ and constant central curvature $C$. Fixing a Kähler class, let $\tilde{g}$ be the unique Calabi-Yau metric with Kähler form in this class whose Ricci form $\tilde{\rho}$ equals $\rho$. Then, in particular, $\operatorname{det} \tilde{\rho}=\operatorname{det} \rho$, and, again by the Calabi-Yau Theorem, $\operatorname{det} \tilde{\omega}=A \operatorname{det} \omega$ for some positive constant $A$. Therefore, the central curvature $\tilde{C}$ of $\tilde{g}$ satisfies $\tilde{C}=\frac{1}{A} C$, and so is constant.

Note also that the sign of $\tilde{C}$ (if nonzero) is the same as that of $C$.

Thus, for example, the Kähler cone of a manifold admitting a Kähler-Einstein metric is completely filled with constant central curvature representatives. Note that there are manifolds that do not admit Kähler-Einstein metrics, but still admit metrics of constant central curvature. For example, generalized Kähler-Einstein metrics in the sense of $\mathrm{Mt}$ (i.e., metrics with eigenvalues of the Ricci tensor constant with respect to the metric) have constant central curvature. The constant central condition is, however, strictly more general than the generalized KählerEinstein one. For example, the general position 3-point blow-up of $\mathbb{C P}^{2}$ admits a Kähler-Einstein metric $\mathrm{Su}$, TY], and it also admits Kähler classes for which $\mathcal{F}_{[\omega]}$ does not vanish identically [LSm. Such a Kähler class admits metrics with constant central curvature by Theorem 9. But these metrics cannot be generalized Kähler-Einstein, since the latter have constant scalar curvature, which forces the vanishing of the Futaki invariant. Nonetheless, it seems at least plausible that every manifold admitting a Kähler metric of constant nonzero central curvature also admits a generalized Kähler-Einstein metric. This is clearly false for metrics with vanishing central curvature.

Turning to uniqueness, the following asymptotic estimates will be employed.

Theorem 10 (Demailly $[\overline{D m}]$ ). Let $L$ be a holomorphic line bundle over a compact complex manifold $M^{n}$, equipped with a smooth Hermitian metric having curvature 
$\rho_{L}$. Denote

$$
\begin{aligned}
M_{\rho_{L}}(q) & :=\left\{m \in M \mid\left(\rho_{L}\right)_{m} \quad \begin{array}{ll}
\text { has } q \text { negative eigenvalues } \\
\text { and }-q \text { positive eigenvalues }
\end{array}\right\} \\
h^{q}(L) & :=\operatorname{dim} h^{q}(M, \mathcal{O}(L)) .
\end{aligned}
$$

The cohomology groups with values in $L^{\otimes k}$ satisfy, for large enough $k$,

$$
h^{q}\left(L^{\otimes k}\right) \leq \frac{k^{n}}{n !} \int_{M_{\rho_{L}}(q)}(-1)^{q}\left(\frac{i}{2 \pi} \rho_{L}\right)^{\wedge n}+o\left(k^{n}\right),
$$

and

$$
\chi\left(L^{\otimes k}\right)=\frac{k^{n}}{n !} \int_{M}\left(\frac{i}{2 \pi} \rho_{L}\right)^{\wedge n}+o\left(k^{n}\right) .
$$

Corollary 8.1. Given the above, if $c_{1}(L)$ admits a positive definite $(1,1)$-form, it does not admit a non-degenerate $(1,1)$-form of any other signature.

Proof of Corollary 8.1. Using the positive definite form $\rho_{L}$, for any $q \geq 1$, the numbers $h^{q}\left(L^{\otimes k}\right)$ have an asymptotic growth rate strictly smaller than $k^{n}$, by (26) (since $\left.M_{\rho_{L}}(q)=\emptyset\right)$. On the other hand, using (27), the Euler characteristic $\chi\left(L^{\otimes k}\right)$ has asymptotic growth equal to $k^{n}$. It follows that $h^{0}\left(L^{\otimes k}\right)$ also has asymptotic growth rate equal to $k^{n}$ (for this part one can also use the proof of the Kodaira Embedding Theorem). Now if there exists another non-degenerate form $\tilde{\rho}_{L}$ in $c_{1}(L)$, with $q_{0}$ negative eigenvalues, and $q_{0}>0$, then by inequality (26) again for $\tilde{\rho}_{L}$, the asymptotic growth of $h^{0}\left(L^{\otimes k}\right)$ must be strictly less than $k^{n}\left(\right.$ since $\left.M_{\tilde{\rho}_{L}}(0)=\emptyset\right)$. This is a contradiction.

This corollary can be considerably strengthened, at least for projective manifolds, for which it can be shown that if a $(1,1)$-class admits a curvature form of constant rank (and, therefore, constant signature), then any other curvature form in this class will have the same rank and signature $\mathrm{Br}$. Using another vanishing theorem, we will prove a relevant special case.

Theorem 11. If a Hermitian line bundle L (over a compact complex manifold) has a curvature form which is negative in one direction at each point, then $L$ admits no nonzero holomorphic sections.

For the proof, see [Kb2 Chapter III, before Corollary 1.16].

Corollary 8.2. If the first Chern class $c_{1}(L)$ of a line bundle over a compact Kähler manifold vanishes, then it does not admit a Hermitian metric with nonzero curvature of constant rank.

Proof. Assuming the contrary, either $c_{1}(L)$ or $c_{1}\left(L^{*}\right)$ admits a curvature 2-form with at least one negative eigen-direction at each point, and by assumption they both admit a flat connection. Thus, by summing curvatures, $c_{1}\left(L \otimes L^{*}\right)$ also admits a curvature 2 -form with at least one negative eigen-direction, and so has no nontrivial holomorphic sections. But the trivial bundle $L \otimes L^{*}$ does admit such sections. This gives a contradiction.

We use Corollaries 8.1 and 8.2 to prove uniqueness results for central metrics of constant central curvature on manifolds for which the first Chern class has a sign. First we show 
Proposition 8.3. Let $M$ be of Fano type. If a central Kähler metric (of either type) with Kähler form representing $c_{1}$ has constant central curvature, then it is Kähler-Einstein.

Proof. Let $g$ have constant central curvature $C$ with Kähler and Ricci forms $\omega, \rho$, respectively, both representing $c_{1}$. Then $\rho^{\wedge n}=C \omega^{\wedge n}$, with $C$ a positive constant (since $c_{1}^{n}>0$, which also implies $C=1$ ). Now $\rho$ cannot have a degeneracy locus, or else $\rho^{\wedge n}$, and therefore $\omega^{\wedge n}$, would have zeros. But then by Corollary 8.1, $\rho$ must be positive definite, since $\omega$ is. Hence by the uniqueness part of the Calabi-Yau theorem, $\rho$ actually equals $\omega$. Thus $g$ is Kähler-Einstein.

Remark 8.4. Note that by Theorem 9 and Proposition 8.3, on a manifold of Fano type, metrics of constant central curvature and Kähler-Einstein metrics must exist concurrently.

Remark 8.5. When $c_{1}<0$, a similar proof using $-\rho$ instead of $\rho$ shows that every Kähler metric of constant central curvature in $-c_{1}$ (or any negative multiple of $c_{1}$ ) is Kähler-Einstein. In the third case, $c_{1}=0$, we use Corollary 8.2 to deduce that any metric of constant (necessarily zero) central curvature and Ricci form of constant rank must be Ricci flat.

Although an analogous version of Proposition 8.3 is valid for extremal metrics as well (see [Ft2 Lemma 2.2.3], or [Ft4]), for central metrics it can be used to deduce a general uniqueness statement.

Theorem 12. Let $M$ be of Fano type. If $M$ admits Kähler metrics of constant central curvature, then every Kähler class admits exactly one $G_{0}$-orbit of such metrics, where $G_{0}$ is the identity component of the group $\operatorname{Aut}(M)$ of biholomorphisms of $M$.

Proof. For $c_{1}>0$, by Theorem 9 the class $c_{1}$ admits such metrics, and they are all Kähler-Einstein by Proposition 8.3. The theorem then follows for this class by the uniqueness result of Bando and Mabuchi BM2]. For an arbitrary Kähler class, by the proof of Theorem 9 the uniqueness part of the Calabi-Yau Theorem 8 and Proposition 8.3 once more, any such metric has the Ricci form of a unique KählerEinstein metric, and so the cardinality of $G_{0}$-orbits in this class has to be the same as the cardinality of $G_{0}$-orbits in $c_{1}$.

Remark 8.6. A similar proof applies when $c_{1}<0$, using the unique Kähler-Einstein metric in $-c_{1}[\mathrm{~A},[\mathrm{Yu}]$. In this case there are no automorphisms; so, in particular, there is a unique metric of constant central curvature in every Kähler class. By the last part of the remark following Proposition 8.3 at least for central metrics for which the Ricci form has constant rank, the case $c_{1}=0$ is really the Ricci flat case, and for the latter the result is known by Theorem 8 .

Demailly's theorem provides a relationship between the degeneracy behaviour of the Ricci form of metrics of constant central curvature, and the numbers

$$
h^{0}\left(M, \mathcal{O}\left(\left(K^{*}\right)^{\otimes k}\right)\right),
$$

where $K^{*}$ is the anticanonical bundle. The above considerations apply similarly to give a result that pertains not just to larger classes of central metrics, but to any metric with non-vanishing central curvature. 
Theorem 13. Let $M_{n}$ be a compact complex manifold with anticanonical line bundle $K^{*}=\Lambda^{n} T^{1,0}$. If $M$ admits a Kähler metric with nowhere-vanishing central curvature, then for a unique integer $q$ with $0 \leq q \leq n$, the numbers $h^{q}\left(M, \mathcal{O}\left(\left(K^{*}\right)^{\otimes k}\right)\right)$ have asymptotic growth $k^{n}$, and all other cohomology groups of $K^{*}$ have slower asymptotic growth rates.

The proof is similar to that of Corollary 8.1. The proof of another vanishing theorem $\mathrm{AG}], \mathrm{BU}$, shows that the other cohomology groups actually vanish asymptotically. Note that the examples of $[\mathrm{HM}]$ of type I central metrics all have nowhere-vanishing central curvature, and this appears to be forced by the topology. On the other hand, for the comparable condition of nowhere-vanishing scalar curvature, one may only conclude an asymptotic growth rate slower than $k^{n}$ for the cohomological dimensions corresponding either to $q=0$, or else to $q=n$.

The above considerations show that perhaps metrics with constant central curvature may be classified. The following partial classification for Kähler surfaces, which was suggested by Claude LeBrun, serves as an illustration.

For a Kähler surface, the Ricci endomorphism is represented locally by a Hermitian matrix with two eigenvalues at each point. When the central curvature is constant, the sign of its determinant is constant throughout the manifold. If it is positive, either both eigenvalues are positive, so $c_{1}>0$, or both are negative and $c_{1}<0$. If $c_{1}>0$ and such a metric exists, there exists another such metric with Kähler form in $c_{1}$, by Theorem 9, The latter is Kähler-Einstein by Proposition 8.3 The Del Pezzo surfaces which admit such metrics are well-known. The Kodaira dimension of these surfaces is $-\infty$. If $c_{1}<0$, the same argument, or Yau's existence theorem for the complex Monge-Ampère equation, implies again that the space admits a Kähler-Einstein metric and is of general type. Its Kodaira dimension is 2 .

The remaining non-degenerate case occurs when the determinant of the Ricci endomorphism is negative, i.e., the eigenvalues of $\rho$ have opposite sign. Such a surface must have Kodaira dimension $-\infty$, by Theorem 11. This eigenvalue structure also shows that the surface admits a semi-definite Kähler metric, and, in the context of a classification of semi-definite Kähler-Einstein metrics, examples are given in [Pt] which are minimal ruled surfaces over a base of genus greater than one. The latter are all projectivizations of quasi-stable bundles.

Finally, the determinant could be zero, which implies that $c_{1}^{2}=0$, and so the surface is not Fano, nor of general type. If one of the eigenvalues is everywhere positive (so for $-\rho$, everywhere negative), the Kodaira dimension is $-\infty$ again by Theorem 11. As examples one can take (certain) ruled surfaces over a base of genus 1. On the other hand, in the remaining cases of Kodaira dimension 0 or 1, the vanishing of $c_{1}^{2}$ implies that the surface is necessarily minimal. By Corollary 8.2 the Kodaira dimension zero case, for which $c_{1}=0$, corresponds, if both eigenvalues are zero, to the Ricci-flat case including tori, K3 surfaces and their quotients. If one eigenvalue is everywhere negative, the Kodaira dimension is one and the surface is elliptic.

Note that classifying $C=0$ metrics with a Ricci endomorphism that changes rank from point to point appears much more difficult.

\section{Existence of Type I Metrics}

It is shown in $\mathrm{HM}$ ] that type I central metrics (with a non-trivial type I central vector field) do, in fact, exist. For example, 
Theorem 14 (Hwang-Maschler [HM]). Real-analytic type I central metrics with non-constant positive central curvature exist on the first Hirzebruch surface $H_{1}:=$ $\mathbb{P}(\mathcal{O}(1) \oplus \mathcal{O}) \rightarrow \mathbb{P}^{1}$

These metrics also possess a positive definite Ricci tensor. Note that all Hirzebruch surfaces $H_{n}$ admit extremal metrics [C1]. At least the construction used in [HM] yields no central metrics on the other Hirzebruch surfaces. The space $H_{1}$ is, however, the only Hirzebruch surface that admits an extremal metric globally conformal to an Einstein metric $[\overline{\mathrm{Dr}}],[\mathrm{Le}],[\mathrm{HS} 2]$. Such a metric has non-constant positive scalar curvature, and this may perhaps be considered a rough extremal analog of Theorem 14. Note, however, that the vanishing theorems of the previous section, along with the assumption of either positive central or scalar curvature, are not sufficiently refined to detect a distinct behaviour among different Hirzebruch surfaces. Finally, on ruled surfaces over a base of negative genus, there are metrics with non-constant negative central curvature, but there appears to be no similar specific statement regarding the sign of the scalar curvature for extremal metrics on such spaces. In fact, the scalar curvature may vanish at some points.

\section{REFERENCES}

[A] T. Aubin, Équations du type Monge-Ampère sur les variétés kählériennes compactes, Bull. Sci. Math. (2) 102 (1978), 63-95. MR 81d:53047

[AG] A. Andreotti and H. Grauert, Théorèmes de finitude pour la cohomologie des espaces complexes, Bull. Soc. Math. France 90 (1962), 193-259. MR 27:343

[B] S. Bando, An obstruction for Chern class forms to be harmonic, unpublished.

[BM1] S. Bando and T. Mabuchi, On some integral invariants on complex manifolds, I. Proc. Japan Acad. Ser. A Math. Sci. 62 (1986), 197-200. MR 88g:58036

[BM2] S. Bando and T. Mabuchi, Uniqueness of Einstein Kähler metrics modulo connected group actions, Algebraic Geometry, Sendai 1985, Advanced Studies in Pure Mathematics 10, North-Holland, Amsterdam-New York, 1987, 11-40. MR 89c:58029

[Bc1] S. Bochner, Vector fields on complex and real manifolds, Ann. of Math. (2) 52 (1950), 642-649. MR 12:2839

[Bc2] S. Bochner, Vector fields and Ricci curvature, Bull. Amer. Math. Soc. 52 (1946), 776-797. MR 8:230a

[BY] S. Bochner and K. Yano, Curvature and Betti Numbers, Annals of Mathematics Studies 32, Princeton University Press, Princeton, N.J., 1953. MR 15:989f

[Bt1] R. Bott, Vector fields and characteristic numbers, Michigan Math J. 14 (1967), 231-244. MR 35:2297

[Bt2] R. Bott, A residue formula for holomorphic vector-fields, J. Differential Geometry 1 (1967), 311-330. MR 38:730

[Br] M. Braverman, private communication.

[BU] D. Borthwick and A. Uribe, Almost Complex Structures and Geometric Quantization, preprint dg-ga/9608006, 1996; Math. Res. Lett. 3 (1996), 845-861; erratum, ibid. 5 (1998), 211-212. MR 98e:58084; MR 99c:58061

[C1] E. Calabi, Extremal Kähler metrics, Seminar on Differential Geometry (S. T. Yau, ed.), Annals of Mathematics Studies 102, Princeton University Press, Princeton, N.J., 1982, 259-290. MR 83i:53088

[C2] E. Calabi, Extremal Kähler metrics II, Differential Geometry and Complex Analysis (I. Chaval and H.M. Farkas, eds.), Springer, Berlin-New York, 1985, 95-114. MR 86h:53067

[Co] H.-D. Cao, Existence of gradient Kähler-Ricci solitons, Elliptic and parabolic methods in geometry (Minneapolis, MN, 1994), 1-16, A. K. Peters, Wellesley, MA, 1996. MR 98a:53058

[Cr] James B. Carrell, A remark on the Grothendieck residue map, Proc. Amer. Math. Soc. 70 (1978), 43-48. MR 58:11528

[Dm] J. P. Demailly, Holomorphic Morse inequalities, Proceedings of Symposia in Pure Mathematics 52, Part 2 (1991), 93-114. MR 93b:32048 
[Dr] A. Derdzinski, Self-dual Kähler manifolds and Einstein manifolds of dimension four, Compositio Math. 49 (1983), 405-433. MR 84h:53060

[Fj] A. Fujiki, On automorphism groups of compact Kähler manifolds, Invent. Math. 44 (1978), 225-258. MR 58:1285

[Ft1] A. Futaki, An obstruction to the existence of Einstein Kähler metrics, Invent. Math. 73 (1983), 437-443. MR 84j:53072

[Ft2] A. Futaki, Kähler-Einstein Metrics and Integral Invariants, Lecture Notes in Mathematics 1314, Springer-Verlag, Berlin and New York 1988. MR 90a:53053

[Ft3] A. Futaki, On compact Kähler manifolds of constant scalar curvature, Proc. Japan Acad., Ser. A, Math. Sci. 59 (1983), 401-402. MR 85i:53070

[Ft4] A. Futaki, Integral invariants in Kähler geometry, Amer. Math. Soc. Transl. Ser. 2, 160 (1994), 63-77. MR 93h:53044 (Japanese original)

[FM] A. Futaki and T. Mabuchi, Bilinear forms and extremal Kähler vector fields associated with Kähler classes, Math. Ann. 301 (1995), 199-210. MR 95m:32039

[FMS] A. Futaki and T. Mabuchi and Y. Sakane, Einstein-Kähler metrics with positive Ricci curvature, Kähler Metrics and Moduli Spaces, Advanced Studies in Pure Mathematics 18-II, Academic Press, Boston, MA, 1990, 11-83. MR 94c:32019

[FR1] A. Futaki and S. Morita, Invariant polynomials on compact complex manifolds, Proc. Japan Acad. Ser. A Math. Sci. 60 (1984), 369-372. MR 86f:32033

[FR2] A. Futaki and S. Morita, Invariant polynomials of the automorphism group of a compact complex manifold, J. Differential Geom. 21 (1985), 135-142. MR 87h:32061

[FT] A. Futaki and K. Tsuboi, Eta invariants and automorphisms of compact complex manifolds, Advanced Studies in Pure Mathematics, 18-I, Academic Press, Boston, MA, 1990, 251-270. MR 93b:58137

[GH] P. Griffiths and J. Harris, Principles of Algebraic Geometry, Pure and Applied Mathematics, Wiley-Interscience [John Wiley \& Sons], New York 1978. MR 80b:14001

[Gu] D. Z.-D. Guan, Quasi-Einstein metrics, Internat. J. Math. 6 (1995), 371-379. MR 96e:53060

[H1] A. D. Hwang, Extremal Kähler metrics and the Calabi energy, Proc. Japan Acad. Ser. A Math. Sci. 71 (1995), 128-129. MR 96e:58035

[H2] A. D. Hwang, On the Calabi energy of extremal Kähler metrics, Internat. J. Math. 6, (1995), 825-830. MR 96i:58032

[H3] A. D. Hwang, On existence of Kähler metrics with constant scalar curvature, Osaka J. Math. 31 (1994), 561-595. MR 96a:53061

[HM] A. D. Hwang and G. Maschler, Central Kähler metrics with non-constant central curvature, Trans. Amer. Math. Soc. 355 (2003), 2183-2203.

[HS1] A. D. Hwang and S. R. Simanca, Distinguished Kähler metrics on Hirzebruch surfaces, Trans. Amer. Math. Soc. 347 (1995), 1013-1021. MR 95e:58045

[HS2] A. D. Hwang and S. R. Simanca, Extremal Kähler metrics on Hirzebruch surfaces which are locally conformally equivalent to Einstein metrics, Math. Ann. 309 (1997), 97-106. MR 98f:58056

[Kb1] S. Kobayashi, Transformation Groups in Differential Geometry, reprint of the 1972 edition, Classics in Mathematics, Springer-Verlag, Berlin, 1995. MR 96c:53040

[Kb2] S. Kobayashi, Differential Geometry of Complex Vector Bundles, Iwanami Shoten, Publishers, and Princeton University Press, 1987. MR 89e:53100

[Kb3] S. Kobayashi, Hyperbolic Manifolds and Holomorphic Mappings, Marcel Dekker, Inc., New York 1970. MR 43:3503

[Ki] N. Koiso, On rotationally symmetric Hamilton's equation for Kähler-Einstein metrics, Recent Topics in Differential and Analytic Geometry, Advanced Studies in Pure Mathematics 18-I, Academic Press, Boston, MA, 1990, 327-337. MR 93d:53057

[La] J. Lafontaine, Courbure de Ricci et fonctionelles critiques, C. R. Acad. Sci. Paris Sér. I Math. 295 (1982), no. 12, 687-690. MR 84g:58035

[Le] C. LeBrun, Einstein metrics on complex surfaces, Geometry and Physics (Aarhus, 1995), Lecture Notes in Pure and Applied Mathematics, 184, Marcel Dekker, New York, 1997, 167-176. MR 97j:53048

[LSm] C. LeBrun and S. R. Simanca, Extremal Kähler metrics and complex deformation theory, Geom. Funct. Anal. 4 (1994), 298-336. MR 95k:58041 
[LSn] C. LeBrun and M. Singer, Existence and deformation theory for scalar-flat Kähler metrics on compact complex surfaces, Invent. Math. 112 (1993), 273-313. MR 94e:53070

[Lc1] A. Lichnerowicz, Isométries et transformations analytiques d'une variété kählérienne compacte, Bull. Soc. Math. France 87 (1959), 427-437. MR 22:5012

[Lc2] A. Lichnerowicz, Variétés kählériennes et première classe de Chern, J. Differential Geom. 1 (1967), 195-223. MR 37:2150

[Lu] K. Liu, Holomorphic equivariant cohomology, Math. Ann. 303 (1995), 125-148. MR 97f:32041

[Mb] T. Mabuchi, An algebraic character associated with the Poisson Brackets, Recent Topics in Differential and Analytic Geometry, Advanced Studies in Pure Mathematics 18-I, Academic Press, Boston, MA, 1990, 339-358. MR 93a:32047

[Ms] G. Maschler, Distinguished Kähler metrics and Equivariant Cohomological Invariants, thesis, State University of New York at Stony Brook, August 1997.

[Mt] Y. Matsushima, Remarks on Kähler-Einstein manifolds, Nagoya Math J. 46 (1972), 161173. MR 46:2615

[Pt] J. Petean, Indefinite Kähler-Einstein metrics on compact complex surfaces, Comm. Math. Phys. 189 (1997), 227-235. MR 98i:32049

[Sm] S. R. Simanca, Precompactness of the Calabi energy, Internat. J. Math. 7 (1996), 245-254. MR 96m:58050

[Su] Y. T. Siu, The existence of Kähler-Einstein metrics on manifolds with positive anticanonical line bundle and a suitable finite symmetry group, Ann. of Math. (2) 127 (1988), 585-627. MR 89e:58032

[T] G. Tian, Kähler-Einstein metrics on algebraic manifolds, Transcendental methods in algebraic geometry (Cetraro, 1994), 143-185, Lecture Notes in Math., 1646, Springer, Berlin, 1996. MR 98j:32035

[Tf] C. W. Tønnesen-Friedman, Extremal Kähler Metrics on Ruled Surfaces, Institut for Matematik og Datalogi Odense Universitet Preprint Nr. 36, 1997.

[Ts1] K. Tsuboi, The lifted Futaki invariants and the Spin ${ }^{c}$-Dirac operators, Osaka J. Math. 32 (1995), 207-225. MR 97a:53109

[Ts2] K. Tsuboi, On the integral invariants of Futaki-Morita and the determinant of elliptic operators, Far East J. Math. Sci. 5 (1997), 305-319. MR 98h:58196

[TY] G. Tian and S. T. Yau, Kähler-Einstein metrics on complex surfaces with $C_{1}>0$, Comm. Math. Phys. 112 (1987), 175-203. MR 88k:32070

[W] E. Witten, Supersymmetry and Morse theory, J. Differential Geom. 17 (1982), 661-692. MR 84b:58111

[Yn] K. Yano, Sur un théorème de M. Matsushima, Nagoya Math J. 12 (1957), 147-150. MR 20:2476

[Yo] M. Yotov, On the generalized Futaki invariant, electronic preprint math/9907055.

[Yu] S. T. Yau, On the Ricci curvature of a compact Kähler manifold and the complex MongeAmpère equation I, Comm. Pure Appl. Math. 31 (1978), 339-411. MR 81d:53045

[Z] W. P. Zhang, A remark on a residue formula of Bott, Acta Math. Sinica (N.S.) 6 (1990), 306-314. MR 91j:58153

Department of Mathematics, Univerity of Toronto, Toronto, Ontario, Canada M5S $3 \mathrm{G} 3$

E-mail address: maschler@math.toronto.edu 